\title{
Dengue outbreak in Bhilai, Chhattisgarh: Entomological investigation and community awareness
}

\section{Raju Ranjha ${ }^{1}$, Himmat Singh ${ }^{2}$, Jitendra Kumar ${ }^{3}$}

${ }^{1}$ Scientist B and Officer-in-Charge, ICMR-National Institute of Malaria Research, Field Unit, Raipur, Chhattisgarh, India; ${ }^{2}$ Scientist D, ICMR-National Institute of Malaria Research, New Delhi, India; ${ }^{3}$ State Programme Officer, National Vector Borne Disease Control Programme, Raipur, Chhattisgarh, India

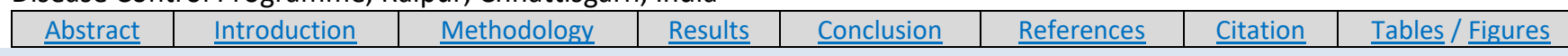

\section{Corresponding Author}

Dr. Raju Ranjha, ICMR-National Institute of Malaria Research, Field Unit, Raipur, Chhattisgarh, India

E Mail ID: dr.ranjha01@gmail.com

\section{Citation}

Ranjha R, Singh $\mathrm{H}$, Kumar J. Dengue outbreak in Bhilai, Chhattisgarh: Entomological investigation and community awareness. Indian J Comm Health. 2021;33(3):512-514. https://doi.org/10.47203/IJCH.2021.v33i03.018

Source of Funding: Nil Conflict of Interest: None declared

\section{Article Cycle}

Received: 08/06/2021; Revision: 03/08/2021; Accepted: 15/08/2021; Published: 30/09/2021

This work is licensed under a Creative Commons Attribution 4.0 International License.

\section{Abstract}

Dengue is the most common arboviral infection worldwide. This study aimed to determine the entomological and communityrelated factors involved in dengue spread during the outbreak in Bhilai, Chhattisgarh, 2018. This was a cross-sectional study. The household survey was done to find out the vector responsible for disease transmission and favourable breeding containers. Susceptibility of larvae towards the larvicide being used was carried out. A questionnaire-based survey was undertaken to find out the awareness of residents in the area towards dengue. House index, Container index, and Breteau index were 92.31, 33.14 and 123.34 , respectively. Larvae were susceptible to the larvicide being used by the local health department. A high number of wet containers available were contributing to high vector breeding. Community awareness was low in the affected area. Water storage practices and conducive climatic conditions lead to increased vector breeding.

\section{Keywords}

Aedes; Community Participation; Dengue; Larva; Mosquito Vectors

\section{Introduction}

Dengue is a most common mosquito-borne viral illness transmitted by female mosquitoes, mainly of the species Aedes aegypti and, to a lesser extent, Ae. albopictus. Dengue is a pandemic-prone viral disease spreading in many parts of the world. Only nine countries had experienced a dengue epidemic before 1970. However, Now the disease is endemic in about 128 countries (1). Dengue cases are reported from almost every state of India. A total of 188401 cases and 325 deaths were reported from dengue in 2017 (2).

There were about 100 dengue outbreaks in the country during 2018 (3). The number of dengue cases surges during the rainy season due to the availability of stagnant freshwater for Aedes breeding. Vector control is one of the best methods to control dengue. Removal of any stagnant water weekly is one intervention to help cut further emergence of adult mosquitoes. As stagnant freshwater may be present in several household containers, community awareness and dengue vector control participation are required.

\section{Aim \& Objective}

To establish the vector responsible for outbreak and the associated factors.

\section{Material \& Methods}

Study Area: This study was carried out at Bhilai, Durg, Chhattisgarh. In July 2018, a Dengue outbreak was reported in Bhilai, Durg, Chhattisgarh. At the request of the state health department, an entomological investigation of the outbreak was carried out. The study was carried out at khursipar, Bhilai. Site selection was made purposely based on the clinically confirmed dengue cases reported and by consulting Local Health authorities. Households were randomly selected within the selected localities. In Bhilai, Chhattisgarh rainy season is from June to October, with peak rainfall in July.

Sample size and inclusion criteria: Sample size for community awareness was calculated based on a previous study in Chhattisgarh(4). At 99\% confidence interval, 5\% 
margin of error, the sample size was estimated to be 200 .

The survey for community awareness included Head of family/adults who were willing to participate in the survey. The migrant population and persons unwilling to participate in the survey were excluded.

Larval Survey and larval susceptibility status: A household-based survey was carried out to assess the dengue situation. All water-holding containers and breeding habitats were inspected. Mosquito immatures were collected, identified using standard identification keys (5) and subjected to larvicidal assays to assess susceptibility towards larvicide being used in outbreak control as per WHO protocol (6). The larval susceptibility test was done on field-collected $3^{\text {rd }}$ and $4^{\text {th }}$ instar larvae. Temephose concentration of $0.02 \mathrm{mg} /$ litre was used to test the Aedes larval susceptibility. Test was carried out at $27 \pm 2{ }^{\circ} \mathrm{C}$ temperature, $75 \pm 10 \%$ relative humidity and low illumination.

Larval indices were calculated as House Index (HI), Container Index $(\mathrm{Cl})$, Breteau Index (BI). The breeding preference ratio (BPR) was calculated to determine the vector's preferential breeding container. It was calculated using the formula, $\mathrm{BPR}=(\%$ of positive Container /Percentage of wet containers) $* 100$

Community awareness survey: Besides, community awareness level on dengue transmission and control was assessed using a questionnaire-based survey. The survey was carried out from $07^{\text {th }}$ August 2018 to $20^{\text {th }}$ August 2018.

Ethical Consideration: The investigation was an outbreak investigation carried out at the request of the State Health Department Chhattisgarh. Research Integrity Committee approved the manuscript, ICMR-National Institute of Malaria Research, New Delhi, Research integrity Committee Number- RIC-03/2021. Written consent was obtained from all the participants involved in the study.

\section{Results}

Larval Indices and preferred breeding habitats: Entomological surveillance was conducted in about 1007 houses, out of which 930 houses were found positive for Aedes breeding. 3748 different containers were checked, of which 1242 containers were found positive for Aedes breeding. The overall $\mathrm{HI}, \mathrm{Cl}$, and $\mathrm{BI}$ were 92.35, 33.14 and 123.34, respectively. Mud pots and Discarded tyres were the containers with the highest Breeding preference ratio. Bucket, Plastic drums and coolers were the most frequent available wet containers in the area (Figure 1).

Plastic drums and coolers were the most frequent positive containers, $24.49 \%$ and $22.41 \%$, respectively. Except for overhead tanks, almost all types of containers were harbouring high breeding. Coolers and plastic drums were the containers with high BPR and were also present in high frequency, making them the most favourable positive containers contributing to the vector breeding (Figure 1). Cement tanka and cement tanks were also critical vector breeding habitat due to their high BPR. Bucket was an important vector breeding habitat despite its low BPR due to its high frequency. Despite their high BPR, mud pots and ground level tanks were not significant for vector breeding due to their low frequency.

Larval susceptibility to larvicide being used: Temephose was being used as a larvicide for Aedes larvae control by the State Health Department. The diagnostic dose exposure $(0.02 \mathrm{mg} /$ litre) of temephose showed that the larval population of Aedes was susceptible.

Disease awareness in the community: The community Awareness survey showed shallow knowledge about dengue transmission in the community. Results of the community awareness survey are given in (Figure 2A). Only $21.2 \%$ of respondents know that mosquito is the cause of dengue transmission. Among them, nearly half of the respondents knew about the breeding of the dengue vector. About $66.6 \%$ of the participants answered correctly about the biting time of the Aedes mosquito.

(Figure 2B): Results of Community awareness survey: $21 \%$ of the responders were aware for Dengue transmission due to mosquito. $48.8 \%$ and $66.6 \%$ of the participants gave the correct answer for the Aedes egglaying and Aedes biting time. 55\% were aware of how one can be protected from dengue. $49.9 \%$ of the participants said that treatment for dengue was not possible. Only $35.5 \%$ of the participants were familiar with dengue symptoms.

\section{Discussion}

Incidence of dengue increase with the increase in rainfall. High rainfall results in an increase in freshwater breeding habitats. It also leads to decreased temperature and increased humidity, favouring outdoor vector survival. The monsoon season in Chhattisgarh is from late June to October. The outbreak was reported in July. The timing of the outbreak indicates that rains may be one of the favourable factors for the outbreak. The area where the outbreak was reported had an irregular water supply, and the residents have water storage practices for household purposes.

All the Aedes larval indices were above the critical levels as per WHO guidelines indices, suggesting high Dengue transmission in the area. Containers with fresh water and Aedes Breeding preference determine a container's contribution in Aedes breeding in any area. Although Mud pots and discarded tyres were having the highest BPR, they were in very low numbers available for aedes egg laying. They are reported to be prominent contributors to dengue breeding but not in this outbreak, due to their low number (7). Plastic drum, cooler, cement tank, cement taka and bucket were the significant contributors constituting about $95 \%$ of the total positive containers. These were having high BPR and available in very high numbers for egg-laying (Figure 1). Earlier studies also reported rain and water storage practices as a vital factor 
for the dengue outbreak. Our study supports earlier observations, water storage practices of residents may be the factor for high larval positive containers (8). The insecticide susceptibility test assessed Larval susceptibility towards Temephose. Our test results showed that the field larvae collected were sensitive to the anti-larval being used in the area.

In developing countries where resources are limited, community-based control programmes involving local people to eliminate breeding sites are an effective and sustainable method to control dengue transmission (9). In our community awareness survey, we found poor knowledge about dengue transmission (Figure 2).

Despite the larvae being susceptible to the larvicidal being used, the larval indices were high. Low awareness in the community about the disease and its transmission may delay outbreak containment. The water storage practices provide ideal space for vector breeding in the area. Buckets that were found to have low BPR in our study were a critical breeding habitat due to the water storage practices in the buckets. The Low community awareness and participation in the control programme is one of the reason for high larval positivity in the containers.

The presence of conducive breeding habitats and favourable climatic conditions due to rains created ideal environment for vector flourishing, further augmented by low community awareness about the disease. This is the first study on assessment community awareness for dengue in this part of Chhattisgarh. However, some studies on another mosquito-borne illness reported insufficient knowledge and the need for health education campaigns to control disease (10). Our study supports the earlier observations that increased focus on health education is required to control the disease. Increased community education campaigns with regular water supply in the area to decrease the water storage practices will be helpful for control of vector-borne disease in the area.

\section{Conclusion \& Recommendation}

Mosquito immature indices were high in the area. High humidity and ambient temperature created a favourable environment for mosquito breeding and led to high disease transmission. The larvicide being used in the area by the local health authorities was effective against the Aedes larvae. Low community awareness may be one of the reasons for delayed control of the outbreak. The present investigation has brought out two significant factors that might be the current situation's significant causes. Firstly, water storage practice: there is a need to change in water storage practices of the inhabitants as a result, there was high breeding. Second lack of awareness: community awareness camps are needed for vectorborne diseases their transmission and control.

\section{References}

1. WHO. Dengue. World Health Organisation; Available from: https://www.who.int/denguecontrol/disease/en/

2. NVBDCP. Dengue/DHF situation in India. In: Programme NVBDC, editor.

3. NCDC. Annual Report In: Control NCfD, editor. 2018.

4. Mendhe H, Bhartendu D, Makade K, Singh D. Awareness and use of Personal Protective Measures against Mosquito Borne Diseases. Indian Journal of Community Health. 2020 09/30;32(3):582 - 5.

5. B.K. Tyagi AMaAV. A catalogue of Indian mosquitoes Indian Journal of Mosquito Research. 2015 13-12-2014;2(2):50-97.

6. WHO. Guidelines for laboratory and field testing of mosquito larvicides. World Health Organization. . 2005.

7. Mahadev PV, Prasad SR, Ilkal MA, Mavale MS, Bedekar SS, Banerjee K. Activity of dengue-2 virus and prevalence of Aedes aegypti in the Chirimiri colliery area, Madhya Pradesh, India. Southeast Asian J Trop Med Public Health. 1997;28(1):126-37.

8. Singh RK, Dhiman RC, Dua VK, Joshi BC. Entomological investigation during an outbreak of dengue fever in Lal Kuan town, Nainital district of Uttarakhand, India. J Vector Borne Dis. 2010;47(3):189-92.

9. Khun SM, Lenore. Community participation and social engagement in the prevention and control of dengue fever in rural Cambodia. Dengue Bulletin. 2008 2008;32:145-55.

10. R. R. A Knowledge, Attitude and Practices Survey and Entomological Situation Analysis in Malaria Endemic Tribal Villages of Surajpur District, Chhattisgarh, India. J Commun Dis. 2019 2019;51(1):1-5.

\section{Figures}

FIGURE 1 WET CONTAINERS

POSITIVE FOR AEDES

BREEDING

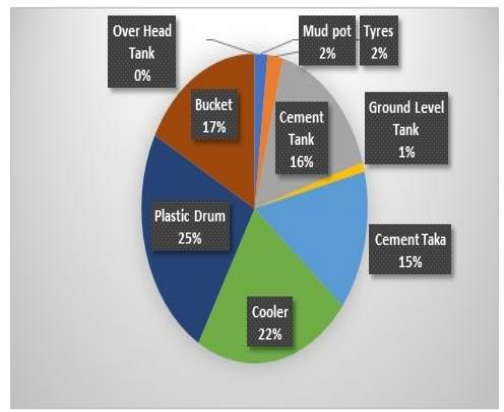

\section{FIGURE 2A KNOWLEDGE

ABOUT DENGUE TRANSMISSION}

= Correct $(\%)=$ Incorrect (\%)

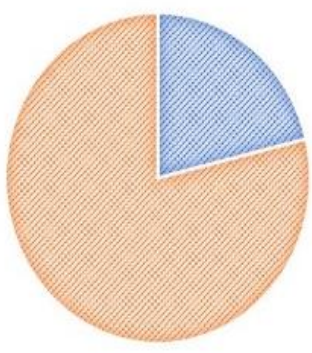

FIGURE 2B BASIC KNOWLEDGE ABOUT DENGUE VECTOR BREEDING AND DISEASE

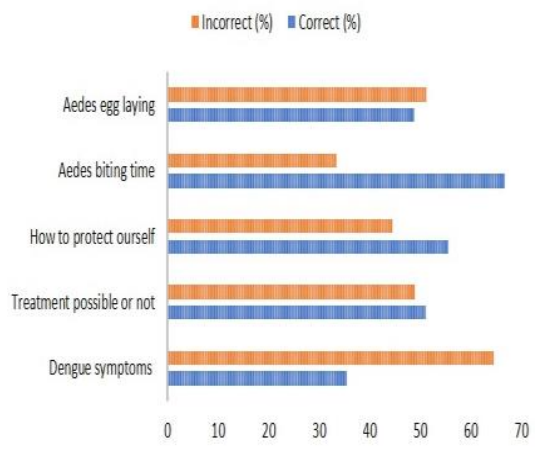

